$\mathrm{E}$

EVALUAR
2019, Vol. 19, No. 1

ISSN 1667-4545

Recuperado de https://revistas.unc.edu.ar/index.php/revaluar

Laboratorio de Evaluación Psicológica y Educativa

Facultad de Psicología - Universidad Nacional de Córdoba

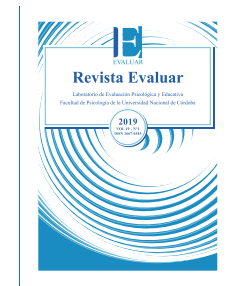

\title{
La evaluación multidimensional de la ansiedad: Técnicas, aplicaciones y alcances
}

\section{The multidimensional evaluation of anxiety: Techniques, applications and scope}

\author{
Iliana Díaz-Kuaik * 1, Guadalupe de la Iglesia ${ }^{2}$ \\ 1 - Facultad de Psicología - Universidad de Buenos Aires, Argentina. \\ 2 - Consejo Nacional de Investigaciones Cientificas y Técnicas - Universidad \\ de Palermo, Universidad de Buenos Aires, Argentina.
}

\author{
Introducción \\ Evaluación de las dimensiones \\ Aplicaciones clínicas de la evaluación \\ multidimensional de la ansiedad \\ Alcances de la evaluación multidimen- \\ sional en investigación clínica \\ Conclusiones \\ Referencias
}

Recibido: 06/09/2018 Revisado: 08/10/2018 Aceptado: 05/11/2018

\section{Resumen}

La ansiedad es un constructo complejo que requiere la valoración de múltiples dimensiones. Conforme fue creciendo el conocimiento sobre los mecanismos implicados en la respuesta de ansiedad, se fue diversificando la tecnología utilizada para su evaluación. El objetivo de este trabajo es realizar un recorrido instrumental por la evaluación de las distintas dimensiones, definidas según la teoría cognitivo-conductual de la ansiedad (Clark \& Beck, 2012). Con tal propósito, se exponen las principales técnicas utilizadas y las propuestas instrumentales generadas por los diferentes modelos teóricos que abordan la ansiedad con sus ventajas y limitaciones. Asimismo, se analizan las aplicaciones clínicas de la evaluación multidimensional de la ansiedad, así como sus alcances en investigación clínica.

Palabras clave: ansiedad, evaluación multidimensional, aplicaciones clínicas, limitaciones

\section{Summary}

Anxiety is a complex construct that requires the assessment of multiple dimensions. As the body of knowledge about the mechanisms involved in the anxiety response increased, the technology used for its evaluation was diversified. The objective of this work is to carry out an instrumental journey through the evaluation of the different dimensions, defined in light of the cognitive behavioral theory of anxiety (Clark \& Beck, 2012). With this purpose, the main techniques used and the instrumental proposals generated by the different theoretical models that address anxiety are exposed along with their advantages and limitations. In addition, the clinical applications of the multidimensional evaluation of anxiety, as well as its scope in clinical research, are analyzed.

Keywords: anxiety, multidimensional evaluation, clinical applications, limitations 


\section{Introducción}

La ansiedad es un constructo complejo abordado por múltiples modelos teóricos que ha evolucionado junto al desarrollo de la disciplina psicológica (De Ansorena-Cao, Cobo-Reinoso, \& Romero-Cagigal, 1983; González-Martínez, 1993; Sierra, Ortega, \& Zubeidat, 2003). Dado que la conceptualización actual de la ansiedad es multidimensional, su operacionalización demanda la valoración de cuatro sistemas de respuesta: fisiológico, cognitivo, conductual y afectivo (Clark \& Beck, 2012; González-Martínez, 1993). La dimensión fisiológica alude a las respuestas defensivas automáticas que preparan al organismo para lidiar con circunstancias aversivas. La dimensión cognitiva refiere al procesamiento cognitivo a través del cual se releva y analiza información relevante sobre la situación, los recursos y el resultado esperado. La dimensión conductual refleja las respuestas conductuales llevadas a cabo para prevenir, controlar, luchar o huir del peligro. La dimensión afectiva alude al malestar subjetivo generado por la experiencia ansiosa (Clark \& Beck, 2012). Para evaluar estas dimensiones se dispone de una amplia variedad de técnicas y propuestas instrumentales generadas por los diversos modelos teóricos. Este trabajo tiene como objetivo describir dichas técnicas y propuestas, sus principales ventajas y limitaciones, considerando las aplicaciones clínicas que posee la evaluación multidimensional y sus alcances en investigación.

\section{Evaluación de la dimensión fisiológica}

La evaluación de las variables fisiológicas de la ansiedad es un legado de los primeros modelos científicos de las emociones aportados por las teorías psicofisiológicas (James 1884, 1890; Lange, 1885). Las técnicas utilizadas en las últimas déca- das difieren sustancialmente de las propuestas instrumentales originales. Sobre todo, porque actualmente existen medidores fisiológicos portables o se prefieren métodos indirectos de evaluación, tales como la observación y los autoinformes, con ciertas ventajas sobre las técnicas objetivas o directas (Buela-Casal \& Sierra, 2004; Calero, Márquez, Vizcarro, \& Fernández-Ballesteros, 2013; Carrobles \& Buela-Casal, 1997).

\section{Evaluación directa de las variables fisiológicas}

La evaluación directa posee una larga tradición de la mano de la psicofisiología y de la psicología experimental, de gran incidencia para el campo de la psicología clínica y de la salud. Ha consistido en la valoración de ciertas respuestas fisiológicas consideradas como índices de procesos o estados psicológicos (Vila, 2004). Los autores de los primeros modelos de las emociones dedicaron especial atención a las respuestas fisiológicas vinculadas a los procesos afectivos y emocionales (James, 1884, 1890; Lange, 1885). Se interesaron en la activación del sistema nervioso autónomo y somático y propusieron la existencia de cierta especificidad fisiológica de las emociones. Posteriormente, se abocaron a los mecanismos neurales centrales de la emoción por la acción combinada de la rama simpática y parasimpática del sistema nervioso autónomo y consideraron que los cambios fisiológicos eran similares para las distintas emociones, con una variación solamente en la intensidad con que se expresaban (Cannon, 1927a, 1927b).

El grado de activación de las variables autonómicas vinculadas con la ansiedad se ha inferido a partir de los valores en la actividad eléctrica de la piel: la conductividad de la piel, y en la actividad cardiovascular: la tasa cardíaca y la presión sanguínea. Las variables somáticas se han infe- 
rido a través de la actividad electromiográfica de los músculos: la actividad eléctrica de los músculos del esqueleto (Carrobles \& Buela-Casal, 1997; Vila, 1998, 2004). Las respuestas fisiológicas más utilizadas para estudiar los mecanismos psicológicos del miedo y la ansiedad proceden del estudio de los reflejos protectores. Estos evitan daños y lesiones, actúan como inhibidores conductuales y facilitan la detección de estímulos amenazantes. Principalmente se han valorado el reflejo de sobresalto y el reflejo cardíaco de defensa. El primero es un patrón de activación motora que se produce por estimulación intensa o aversiva de inicio repentino. Es una reacción refleja, innata, involuntaria y sujeta a condicionamiento con tres componentes principales: parpadeo, inclinación brusca de la cabeza hacia adelante y una onda de flexión que se trasmite desde el tronco hacia las rodillas (Landis \& Hunt, 1939). Se mide a partir del registro psicofisiológico del parpadeo (Lang, Bradley, \& Cuthbert, 1997). El segundo alude al componente cardíaco de la respuesta ante estimulación discreta intensa o aversiva. Se mide a través del cambio en la tasa cardíaca (latidos por minuto) durante los 80 segundos posteriores a la presentación del estímulo (Vila, 1998).

El registro de las variables psicofisiológicas requiere de una serie de fases: (a) detección de la señal proveniente del organismo; (b) transformación de las señales del organismo a señales eléctricas; (c) amplificación de dichas señales; (d) registro propiamente dicho: gráfico, digital y/o computarizado y (e) conversión de la señal registrada para su análisis estadístico (Calero et al., 2013; Carrobles \& Buela-Casal, 1997). Dicho procedimiento ha reportado diversas fuentes de error procedentes de las condiciones físicas del ambiente, la calidad del instrumental, el nivel previo de activación, la discriminación de respuestas no relacionadas con la estimulación, la fluctuación temporal de las variables fisiológicas y los patrones atribuibles a diferencias personales no generalizables, entre otras. Asimismo, la artificialidad de la situación dificulta la inducción de estados emocionales y limita la transferencia y generalización de los resultados. No obstante, actualmente existen medidores portables que permiten la evaluación de las variables fisiológicas en situaciones naturales, lo que ha posibilitado su aplicación en numerosos campos: psicología clínica, medicina conductual, neuropsicología, ergonomía y deporte, entre otros (Calero et al., 2013; Carrobles \& Buela-Casal, 1997).

Evaluación indirecta de las variables fisiológicas

La evaluación indirecta surgió como una opción válida cuando no se contaba con el equipamiento técnico o profesional requerido o cuando por cuestiones de diseño se optaba por modelos no experimentales o clínicos (Carrobles \& Buela-Casal, 1997). Fundamentalmente, se ha realizado a través de técnicas de observación y técnicas de autoinforme. Estas últimas pueden clasificarse según Fernández-Ballesteros (2013c) en: autorregistros, escalas/inventarios/cuestionarios y entrevistas.

La evaluación a través de la observación es posible sólo cuando existen manifestaciones externas fácilmente discriminables. Supone un procedimiento sistemático de recolección de información a partir de la percepción deliberada, por parte del observador entrenado, de ciertas manifestaciones emitidas por un sujeto (Fernández-Ba1lesteros, 2013b). Las unidades de análisis en este caso han sido las manifestaciones externas de las variables psicofisiológicas definidas previamente.

El autorregistro se ha utilizado principalmente para el registro de los síntomas asociados a determinados estados fisiológicos de fácil detección para el sujeto. No se ha utilizado para la 
evaluación del estado fisiológico en sí mismo porque este no es susceptible de evaluación a través de este procedimiento. Su detección en variables como tasa cardíaca, actividad electrodérmica y ondas cerebrales ha podido mejorarse con el entrenamiento y con la utilización de métodos de registro adecuados y/o portables en situaciones naturales (Buela-Casal \& Sierra, 2004; Carrobles \& Buela-Casal, 1997).

Las escalas, cuestionarios o inventarios han sido utilizados para evaluar de forma específica o general una serie de manifestaciones o síntomas asociados a los estados psicofisiológicos de las emociones, tales como el miedo y la ansiedad. Requieren, al igual que el autorregistro, que el sujeto posea la capacidad de discriminación y detección de una manifestación propia. Los instrumentos específicos más utilizados han sido: (a) Cuestionario sobre Percepción Autonómica (APQ; Mandler, Mandler, \& Ulville, 1958), (b) Inventario de Indicadores Psicofisiológicos (Cautela \& Upper, 1976) y (c) Inventario de Ansiedad E-R (Endler, Hunt, \& Rosenstein, 1962). Por su parte, los instrumentos generales para evaluar síntomas clínicos de ansiedad, que incluyen síntomas psicofisiológicos han sido: (a) Escala de Hamilton (HARS; Hamilton, 1959); (b) Inventario de Ansiedad de Beck (BAI; Beck, Epstein, Brown, \& Steer, 1988); (c) Inventario Revisado de 90 síntomas (SCL-R-90; Derogatis, 1977); (d) Inventario de Situaciones y Respuestas de Ansiedad (ISRA; Miguel-Tobal \& Cano-Vindel, 1986); (e) Escala de Ansiedad de Zung (EAZ; Zung, 1971); (f) Escala de Ansiedad y Depresión Hospitalaria (HADS; Zigmond \& Snaith, 1983); (g) Escala de Ansiedad Clínica (CAS; Snaith, Baugh, Clayden, Husain, \& Sipple, 1982); (h) Escala Breve de Ansiedad de Tyrer (BSA; Tyrer, Owen, \& Cicchetti, 1984) e (i) Cuestionario de Detección de Ansiedad (ASQ-15; Wittchen \& Boyer, 1998), entre otros.
Finalmente, la entrevista también ha permitido relevar la presencia y magnitud de los síntomas asociados a los estados psicofisiológicos de la ansiedad. Construidos para aumentar la confiabilidad y validez diagnóstica a través de la estandarización del proceso de evaluación, los criterios internacionales y la indagación sistemática y exhaustiva de síntomas han permitido la inclusión confiable de los pacientes dentro de las categorías diagnósticas y el diagnóstico diferencial en los cuadros limítrofes (Echeburúa, 1996). Específicamente para la evaluación de los trastornos de ansiedad, las entrevistas más utilizadas han sido: (a) Entrevista para los Trastornos de Ansiedad según el DSM-IV (ADIS-IV; Brown, Di Nardo, \& Barlow, 1994), la cual es una entrevista semiestructurada heteroaplicada con la que se realiza una evaluación de los trastornos de ansiedad y sus trastornos comórbidos más comunes; (b) Entrevista Clínica Estructurada para el DSM-IV y DSM-5 (SCID-IV y SCID-5; First, Spitzer, Gibbon, Hilsenroth, \& Segal, 2004, 2015), la cual es una entrevista semiestructurada destinada a realizar los diagnósticos más importantes del eje I del DSM-IV y los diagnósticos equivalentes del DSM-5; (c) Mini Entrevista Neuropsiquiátrica Internacional (MINI; Lecrubier et al., 1997), la cual es una entrevista breve pero de gran difusión que explora los principales trastornos psiquiátricos del eje I del DSM-IV y del CIE-10.

\section{Evaluación de la dimensión conductual}

La conducta es una de las variables más estudiadas y evaluadas en la historia de la psicología. Cada modelo teórico conceptualizó y abordó la conducta desde una perspectiva distinta, diversificándose las estrategias y técnicas utilizadas para su evaluación. Por ejemplo, en términos generales, el modelo psicoanalítico consideró la 
conducta como un signo que necesitaba ser interpretado simbólicamente para adquirir un valor diagnóstico. Las conductas manifiestas, actos fallidos, sueños o ejecución en técnicas proyectivas fueron considerados como el resultado de factores intrapsíquicos, principal objeto de estudio de dicho modelo. Las técnicas proyectivas han sido instrumentos sensibles para revelar aspectos inconscientes de la conducta a partir de una variedad de respuestas subjetivas (Calero et al., 2013). Por su parte, para el modelo psicométrico de rasgos, la conducta también estaba determinada por los atributos intrapsíquicos (llamados rasgos, habilidades o intereses). Al igual que en el modelo psicodinámico, dichas variables no podían evaluarse de forma directa, sino a través de la conducta manifiesta. Esta evaluación requería, en este caso, de una interpretación estadística. Utilizaron tests estandarizados construidos según estrategias teórico racionales, empíricas y factoriales, apoyadas en el supuesto de generalidad o estabilidad de las conductas (Buela-Casal \& Sierra, 1997; Calero \& Padilla, 2013; Fernández-Ballesteros, 2013a).

Por el contrario, el modelo conductual planteó la especificidad situacional de la conducta y, por ende, su dependencia de las variables exógenas o ambientales. Consideró la evaluación como un componente del propio proceso terapéutico, inseparable de la modificación conductual (Buela-Casal \& Sierra, 1997; Fernández-Ballesteros, 1994, 2013a). Incluyó distintos enfoques de evaluación producto de su desarrollo conceptual, metodológico y de la diversificación de sus variables de estudio. Por ejemplo, el modelo clásico tomó como objeto de estudio las respuestas motoras y los estímulos físicos subyacentes y el modelo mediacional incorporó la aplicación de los procesos de aprendizaje abocándose al análisis aplicado de la conducta en función de sus variables situacionales. Consideró la evaluación y la intervención como parte de un mismo proceso destinado a la modificación de la conducta. Si bien no incluyó los procesos fisiológicos ni cognitivos como objeto de estudio, tampoco negó su existencia considerándolos productos de la conducta. Por su parte, el modelo conductual interactivo integró las relaciones entre las variables fisiológicas, cognitivas, situacionales y conductuales, a las que incluyó los estados afectivos o emocionales. Consideró que la conducta podía ser causada o activada por dichos factores aislados o combinados (Buela-Casal \& Sierra, 1997; Fernández-Ballesteros, 2013a).

La evaluación conductual se ha definido como la identificación de unidades de respuesta significativas y sus variables generadoras con el fin de comprender y modificar la conducta (Hayes, Nelson, \& Jarrett, 1986). Supone que el comportamiento puede ser funcionalmente explicado a través de ciertas condiciones ambientales actuales que controlan o mantienen dichas conductas y por ciertas variables personales constituidas a partir de condiciones ambientales pasadas. Estas últimas, en interacción con el organismo, conformarían la personalidad del sujeto. Es un proceso que implica la aplicación de distintos procedimientos de evaluación a través de una serie de fases que requieren cada vez de instrumentos más estrictos, sofisticados y precisos (Fernández-Ballesteros, 1994). A pesar de su diversidad, a grandes rasgos pueden clasificarse en métodos directos y métodos indirectos (Barrios, 1988). Los primeros aluden a los métodos conductuales clásicos que evalúan la conducta en el momento y en el lugar de su ocurrencia real. Los segundos, a métodos que lo hacen en otro tiempo y/o lugar.

\section{Evaluación directa de las variables conductuales}

La evaluación directa de las variables conductuales se ha realizado principalmente a través del registro de la actividad motora y la observa- 
ción sistemática. La actividad motora se evalúa a través del registro instrumental del movimiento en el espacio y la aceleración, lo que ha permitido obtener muestras de la actividad motora naturales, objetivas, confiables, unidimensionales, estables y válidas en condiciones de laboratorio. Sin embargo, requiere equipamiento y profesionales capacitados (Tryon, 1997). A pesar de la difusión que tuvieron en su momento, su uso en la práctica clínica fue limitado, ya que se preferían técnicas creadas específicamente para el registro de la conducta, como la observación o los autoinformes. Sin embargo, con el surgimiento de medidores portables, en la última década se ha podido retomar la evaluación objetiva de las variables fisiológicas en situaciones naturales (Buela-Casal \& Sierra, 2004; Calero et al., 2013).

La observación sistemática ha sido la técnica principal y más eficaz para registrar las conductas motoras de ansiedad. Permite cuantificar la conducta espontánea del sujeto en situaciones naturales en el momento de su ocurrencia. Implica la percepción deliberada, el registro, la codificación y el análisis de dicha conducta a partir de un procedimiento sistematizado que permite la replicabilidad y el control de los resultados. Su objetivo principal es registrar medidas de conducta que posean variabilidad en un intervalo de tiempo determinado. Las unidades de medida más utilizadas han sido: ocurrencia, orden, frecuencia y duración. Requiere de un proceso riguroso de codificación y categorización que posibilite la sistematización de los datos recabados y garantice la precisión de los resultados obtenidos. La incorporación de dispositivos portátiles de registro ha facilitado dicha tarea (Buela-Casal \& Sierra, 2004). Sin embargo, esta técnica ha reportado diversas fuentes de error procedentes de la reactividad del sujeto, del grado de participación del observador, sus expectativas y entrenamiento, y del sistema de observación (tipo de registro elegido, procedimientos y dispo- sitivos técnicos; Fernández-Ballesteros, 2013b; Quera \& Behar, 1997).

\section{Evaluación indirecta}

de las variables conductuales

La evaluación indirecta de la conducta se fue adoptando de forma progresiva en la práctica clínica y de investigación conductual. En las últimas décadas los autoinformes (entrevistas, autorregistros, escalas, cuestionarios e inventarios) han sido las técnicas de evaluación más utilizadas (Buela-Casal \& Sierra, 2004; Fernández-Ballesteros, 1994, 2013c; Jensen, 1996). En este contexto, han adquirido ciertas características distintivas al ser considerados como una muestra de un tipo determinado de conducta y estar relacionados con situaciones específicas (Del Barrio, 1998; Fernández-Ballesteros, 2013c).

En la práctica clínica se han utilizado dos modelos de entrevista: diagnóstica y conductual. La primera busca la exploración diagnóstica y psicopatológica de los trastornos clínicos de acuerdo con los criterios internacionales (Sierra, Buela-Casal, \& Fernández, 2004). Remite a una información general sobre un trastorno, su etiología, desarrollo típico, pronóstico y tratamiento existentes. Dicha información se generaliza a través de diferencias individuales, lo que orienta la práctica e investigación clínica (Vizcarro, 1998). La evaluación clínica requiere, adicionalmente, una evaluación ideográfica más o menos detallada en la búsqueda de leyes particulares que complemente la información general (Fernández-Ba1lesteros, 2013a; Silva, 1998). Las entrevistas estandarizadas ya citadas incluyen la evaluación del comportamiento y sus alteraciones, información crucial para determinar la adaptación y el deterioro funcional del sujeto en áreas importantes de su vida, requisito necesario para diagnosticar 
un trastorno mental (American Psychiatric Association, 2013). Por su parte, la entrevista conductual es una herramienta esencial e indispensable del proceso de evaluación y tratamiento. Pretende relevar información detallada y precisa sobre las conductas problema, los contextos de desarrollo y las consecuencias funcionales y adaptativas para el sujeto. Permite establecer prioridades de intervención y proporciona una dirección operacionalizada para la intervención terapéutica destinada a la modificación de la conducta problema (Buela-Casal \& Sierra, 2004; Bruch \& Meyer, 1996). En la actualidad, tal como en décadas anteriores, la entrevista continúa siendo la técnica más utilizada en la práctica clínica como instrumento de evaluación e intervención psicológica (Márquez, 2013; Sierra et al., 2004).

El autorregistro implica un procedimiento combinado de la técnica de observación y el registro de ciertos aspectos del propio comportamiento como son: ocurrencia, duración, intensidad, antecedentes, consecuentes y resultado. Aporta datos a la evaluación conductual y promueve el cambio (Fernández-Ballesteros, 2013b, 2013c). Es el único método que permite la evaluación de las interacciones en la vida diaria del paciente, la detección de los elementos situacionales y las respuestas más significativas. Sin embargo, esta técnica ha planteado limitaciones en individuos defensivos o con baja capacidad de introspección (Buela-Casal \& Sierra, 2004; Echeburúa, 1996).

Por otro lado, las escalas, cuestionarios e inventarios han cobrado un papel importante en la evaluación conductual actual y son utilizados como instrumentos primordiales para precisar la información recogida con técnicas de amplio espectro como la entrevista, la observación sistemática, la autobiografía, entre otras. Son instrumentos complementarios que, si bien ofrecen escasa especificidad situacional y de respuesta (Buela-Casal \& Sierra, 2004), permiten compa- rar los comportamientos del sujeto con su grupo normativo (Fernández-Ballesteros, 1994, 2013b; Jensen, 1996). Particularmente en la evaluación clínica de los trastornos de ansiedad, el autoinforme ha sido la técnica más utilizada por los evaluadores y terapeutas conductuales (Buela-Casal \& Sierra, 2004; Menéndez-Carrillo \& Maciá-Antón, 1994). A pesar de su difusión, son pocas las escalas que incluyen enunciados con manifestaciones conductuales de ansiedad. De las antes mencionadas, solo el ISRA (Miguel-Tobal \& Cano-Vindel, 1986), incorpora dicha dimensión, a la que denomina respuestas motoras.

\section{Evaluación de la dimensión cognitiva}

Las variables cognitivas de la ansiedad comenzaron a evaluarse a partir del desarrollo de la teoría cognitivo-conductual. Esta consideró a los procesos cognitivos que mediaban entre un estímulo aversivo y la respuesta emocional del sujeto como responsables del desarrollo y mantenimiento de la ansiedad. La valoración cognitiva de sujetos con trastornos de ansiedad permitió comprender su etiología y persistencia (Clark \& Beck, 2012), planificar el tratamiento clínico y asegurar su éxito (Mohlman \& Gorman, 2005). Junto con la formulación cognitiva del caso, se transformaron en pilares primordiales para una psicoterapia efectiva, sirviendo de nexo entre la teoría cognitivo-conductual a y el tratamiento (Clark \& Beck, 2012).

Las variables cognitivas operacionales y de contenido han sido el principal objeto de estudio para este modelo (Buela-Casal \& Sierra, 1997; Fernández-Ballesteros, 2013a). Las primeras aluden a los mecanismos mediante los cuales el sistema opera procesando la información: memoria, atención, codificación, entre otros. Las segundas refieren a los contenidos de la información y sus 
representaciones en proposiciones, pensamientos, imágenes, esquemas, etc. El grado de accesibilidad de lo cognitivo es lo que ha determinado el método idóneo para acceder a dichas variables (Pérez-Pareja, 1997); en la evaluación de los contenidos cognitivos se han preferido los autoinformes, y en la evaluación de las operaciones cognitivas, las técnicas objetivas.

\section{Evaluación de los contenidos cognitivos}

Según Fernández-Ballesteros (2013c), el autoinforme (entrevista, autorregistro, escala/cuestionario/inventario) es el método prioritario y directo por excelencia para explorar los contenidos cognitivos. Es la única vía de acceso a las manifestaciones internas del sujeto, expresadas a través de un mensaje verbal, producto de la introspección o la autoobservación. Los contenidos y productos cognitivos son accesibles a nivel de la conciencia e informables a través de dicha verbalización. La entrevista ha sido la técnica de autoinforme más utilizada para evaluar los contenidos cognitivos (Fernández-Ballesteros, 2013c; Silva, 1998). Las tres entrevistas estandarizadas antes mencionadas para valorar la presencia y severidad de los síntomas típicos de los trastornos de ansiedad de acuerdo con los criterios diagnósticos internacionales también han sido utilizadas para evaluar la dimensión cognitiva, sobre todo a nivel de pensamientos automáticos, imágenes intrusivas e inferencias. Sin embargo, su utilización suele ser engorrosa, demanda mucho tiempo de aplicación y una sólida formación clínica del entrevistador (Buela-Casal \& Sierra, 2004).

Como alternativa a esta, el autorregistro ha sido la segunda alternativa para la evaluación de las cogniciones y el sesgo cognitivo a nivel de pensamientos, especialmente en la clínica e investigación bajo el modelo cognitivo. Su sencillez y aplicabilidad en situaciones naturales han favorecido su utilización. Supone, por un lado, atender de forma deliberada a las cogniciones en el momento en que estas se producen (automonitoreo) $\mathrm{y}$, por otro, su registro mediante un procedimiento preestablecido (Buela-Casal \& Sierra, 2004; Fernández-Ballesteros, 2013c). Esto le permite al paciente instaurar el procesamiento cognitivo reflexivo como respuesta mediadora a situaciones donde primaba el procesamiento cognitivo automático (Clark \& Beck, 2012). Al admitir el registro de varios aspectos a la vez permite en la clínica cognitiva relevar y evaluar variables de desarrollo y mantenimiento de la ansiedad "en tiempo real" (Clark \& Beck, 2012, p. 151). Los autorregistros más utilizados en este ámbito han sido: (a) registros de pensamientos automáticos, (b) valoración diaria de ansiedad y registro de situaciones, (c) registro de pensamientos aprensivos para automonitoreo y (d) registro de valoración, entre otros. Los mismos fueron incluidos por Clark y Beck (2012) en los protocolos de evaluación y tratamiento que validaron. No obstante, existen algunos elementos que han afectado la validez de este procedimiento, por ejemplo, la motivación, la reactividad, la falta de conciencia de las conductas implicadas, la complejidad de la interacción de las respuestas, su grado de accesibilidad, las imprecisiones en el recuerdo o en la comprensión de la situación, la complejidad o inadecuación del formato de registro (Buela-Casal \& Sierra, 2004; Echeburúa, 1996).

Las escalas, cuestionarios e inventarios han sido la tercera modalidad utilizada para evaluar los contenidos cognitivos y las distorsiones cognitivas. Las más difundidas son: (a) Listado de Comprobación de Cogniciones (CCL; Beck, Brown, Steer, Eidelson, \& Riskind, 1987), incluye una subescala de ansiedad (CCL-A) que evalúa la frecuencia de pensamientos ansiosos negativos en torno a temas de incertidumbre y orientación 
hacia el futuro; (b) Cuestionario de Estados de Preocupación Penn (PSWQ; Meyer, Miller, Metzger, \& Borkovec, 1990), evalúa la propensión a la preocupación y la intensidad con que se presentan las experiencias de preocupación, sin hacer referencia a temas específicos; (c) Cuestionario de Pensamientos Automáticos (ATQ; Hollon \& Kendall, 1980), evalúa pensamientos irracionales asociados a la depresión y la ansiedad y (d) Cuestionario de Esquemas Maladaptativos Tempranos (YSQ L2; Young, 1999), evalúa la presencia de dieciséis esquemas disfuncionales desarrollados durante la infancia, estables y duraderos a lo largo de la vida de un individuo, que sirven como marcos para el procesamiento de experiencias posteriores. Asimismo, dentro de las escalas antes mencionadas para evaluar síntomas clínicos generales de ansiedad, las siguientes incluyen información sobre el dominio cognitivo: (a) Inventario de Situaciones y Respuestas de Ansiedad (ISRA; Miguel-Tobal \& Cano-Vindel, 1986); (b) Inventario de Ansiedad Estado Rasgo (STAI; Spielberger, Gorsuch, \& Lushene, 1983); (c) Listado Revisado de 90 síntomas (SCL-R-90; Derogatis, 1977); (d) Inventario de Ansiedad de Beck (BAI; Beck et al., 1988); (e) Escala de Ansiedad de Zung (EAZ; Zung, 1971); (f) Escala de Ansiedad de Hamilton Autoaplicada (HARS; Hamilton, 1959); (g) Escala de Ansiedad y Depresión Hospitalaria (HADS; Zigmond \& Snaith, 1983); (h) Escala de Ansiedad Clínica (CAS; Snaith et al., 1982); (i) Escala Breve de Ansiedad de Tyrer (BSA; Tyrer et al., 1984) y (j) Cuestionario de Detección de Ansiedad (ASQ-15; Wittchen \& Boyer, 1998).

Evaluación de las operaciones cognitivas

Implican procesos secuenciales de procesamiento de la información que no son transmisibles verbalmente por el sujeto, principalmente porque se producen de forma automática y no son accesibles a la conciencia, y secundariamente porque son muy reactivos a la introspección que implica el autoinforme. Por ello, para su evaluación se ha preferido la utilización de técnicas objetivas, como el tiempo de reacción y la respuesta a serie de palabras, entre otras, que admiten la manipulación de los estímulos y el registro automático de las respuestas en base al número de aciertos y errores, según ciertos parámetros temporales $(\mathrm{Ca}-$ lero et al., 2013). Las operaciones cognitivas más evaluadas en los trastornos de ansiedad han sido la codificación y recuperación de la información, la velocidad de procesamiento, la atención selectiva, la atención autofocalizada y la propagación de la activación (Clark \& Beck, 2012). La evaluación del funcionamiento atencional ha ocupado un lugar preponderante; los mecanismos considerados para explicar los sesgos atencionales han sido múltiples, implementados por diferentes operaciones cognitivas (Eysenck, 1988; Eysenck, Derakshan, Santos, \& Calvo, 2007). La tarea más utilizada en investigación experimental para evaluar los sesgos atencionales en sujetos ansiosos ha sido la Stroop emocional, que permitió detectar una lentificación de las respuestas al nombrar el color de palabras específicas ansiógenas, en comparación con las palabras neutrales (Mathews \& MacLeod, 1985). Sin embargo, dicho hallazgo presenta limitaciones interpretativas, no se puede asegurar fehacientemente que la denominación más lenta de la palabra sea producto de un efecto de interferencia por el significado que posee (Clark \& Beck, 2012) ni consecuencia de un sesgo atencional selectivo hacia los contenidos amenazantes (Pacheco-Unguetti, Lupiáñez, \& Acosta, 2009). 


\section{Evaluación de la dimensión afectiva}

La dimensión afectiva comenzó a considerarse y valorarse con el desarrollo de la teoría cognitivo-conductual. Para Clark y Beck (2012), esta derivaba de la activación conjunta de las dimensiones fisiológica y cognitiva y constituía la experiencia subjetiva de la sensación ansiosa, emergente de la valoración cognitiva que el sujeto realizaba principalmente de sus manifestaciones de ansiedad. A pesar de que algunos percibían como perturbadores o amenazantes los síntomas fisiológicos, otros, los fenómenos cognitivos (preocupación o pensamientos intrusos indeseados), y otros, el aumento de la sensación generalizada de ansiedad, la experiencia subjetiva de sentir ansiedad era lo que la persona consideraba como amenazante e intolerable y a la cual reaccionaba de forma defensiva a través de las conductas evitativas (Clark \& Beck, 2012).

Para la teoría cognitivo-conductual, la experiencia subjetiva de ansiedad es la que motiva a las personas a la consulta clínica. Su eliminación ha sido el principal criterio para determinar el éxito del tratamiento, cuyo primer objetivo de intervención se propone normalizar la experiencia de ansiedad. Dado que el paciente se ve compelido a consultar por la perturbación que le produce dicha experiencia ansiosa, evaluarla y abordarla permite aumentar la motivación para el cambio y propiciar la alianza terapéutica. Según Clark y Beck (2012), los pacientes solían mostrarse más dispuestos a aceptar el tratamiento si sus experiencias ansiosas eran abordadas con inmediata relevancia. Por ende, la evaluación de la dimensión afectiva resulta primordial para el diagnóstico, la alianza terapéutica y la eficacia del tratamiento en los trastornos de ansiedad.

Como se mencionó con anterioridad, para Fernández-Ballesteros (2013c) el autoinforme es el procedimiento más directo y prioritario para evaluar los contenidos mentales. Dentro de este, las entrevistas estandarizadas ya citadas incluyen pautas para la valoración de las manifestaciones afectivas, por lo que también han sido utilizadas para evaluar esta dimensión. Por su parte, los autorregistros permiten evaluar la experiencia subjetiva de ansiedad a partir del registro de las situaciones, cogniciones y respuestas de ansiedad que convergen simultáneamente en una situación de interacción. Esta información facilita determinar si la experiencia ansiosa es excesiva o desproporcionada teniendo en cuenta los factores situacionales, culturales y evolutivos. Asimismo, se ha extendido el uso de escalas para evaluar el componente afectivo de los trastornos emocionales. A partir del modelo bidimensional del afecto propuesto por Watson y Clark (1984) sobre la estructura básica del afecto, se desarrolló una escala específica para evaluar la afectividad: la Escala de Afecto Positivo y Negativo (PANAS; Watson, Clark, \& Tellegen, 1988). Watson y Clark (1984) diferenciaron dos dimensiones relativamente independientes del afecto, denominadas afecto positivo y afecto negativo. El afecto positivo representa la dimensión de emocionalidad placentera, caracterizada por la motivación, energía, concentración, alerta y participación agradable. En contraste, el afecto negativo representa la dimensión de emocionalidad displacentera, caracterizada por el malestar, el desinterés subjetivo y la participación desagradable e incluye una variedad de estados emocionales aversivos como el disgusto, la ira, el miedo, la culpa y el nerviosismo (Watson et al., 1988). Este modelo planteó importantes implicaciones en los trastornos de ansiedad y del estado de ánimo al describir características afectivas que son propias de cada cuadro. En la depresión hallaron baja afectividad positiva y alta afectividad negativa, mientras que en la ansiedad, elevada hiperactivación fisiológica y alta afectividad negativa. Por ende, ambas poseen un componente 
común (el afecto negativo), y componentes específicos (bajo afecto positivo y alta hiperactivación fisiológica, respectivamente).

\section{Aplicaciones clínicas de la evaluación multidimensional de la ansiedad}

La evaluación dimensional de la ansiedad proporciona un marco para comprender cómo se manifiesta un trastorno en un paciente determinado y los factores que mantienen la conducta disfuncional. Dicha información resulta beneficiosa para la conceptualización del caso, guía al terapeuta en la conducción de tratamientos eficaces al permitir la planificación de intervenciones efectivas (Beck, 2000; Clark \& Beck, 2012; Keegan $\&$ Holas, 2010). De acuerdo con la evidencia empírica, se emplea un tipo de intervención cuando predominan los síntomas cognitivos y afectivos $\mathrm{y}$ otro distinto cuando predominan los síntomas fisiológicos o conductuales. Por ejemplo, las investigaciones de Lang, Melamed y Hart (1970) y Wolpe (1977) encontraron que la técnica de desensibilización sistemática era más efectiva para tratar los síntomas fisiológicos que los cognitivos. Por su parte, otros autores (Öst, Jerremalm, \& Johansson, 1981; Öst, Johansson \& Jerremalm, 1982) encontraron que aquellos que presentaban mayor reactividad fisiológica se beneficiaban con técnicas de relajación, mientras que aquellos con mayor reactividad motora lo hacían con técnicas de entrenamiento en habilidades sociales. Clark y Beck (2012), a partir de la evidencia acumulada, clasificaron las intervenciones en cognitivas y conductuales. Las primeras fueron utilizadas tanto para tratar los síntomas cognitivos como afectivos, incrementan la flexibilidad cognitiva a través del tratamiento de los pensamientos, valoraciones y creencias ansiosas. Poseen como objetivo primordial cambiar el foco de la amenaza, modificar la amenaza, la vulnerabilidad y las creencias sesgadas, normalizar el miedo y la ansiedad, fortalecer la eficacia personal y resolver los problemas relacionados con la búsqueda de seguridad. Por su parte, las intervenciones conductuales se han utilizado para modificar los patrones de respuesta conductual disfuncionales del paciente, así como también para aliviar y tratar pacientes con gran activación de respuestas fisiológicas. Sin embargo, cabe acotar que para la teoría cognitivo conductual, la cognición, la emoción y la conducta son subsistemas interdependientes. La modificación de un subsistema produce indefectiblemente cambios en los otros dos. Asimismo, un cambio en la respuesta conductual o fisiológica requiere de la intervención sobre los sesgos cognitivos asociados que las generan o sostienen.

La eficacia terapéutica se establece a través del éxito del plan de tratamiento, o sea, del logro de los objetivos propuestos y del efecto esperado (Castro-Solano, 2003; Gómez-Penedo \& Roussos, 2012; Bados-López, García-Grau, \& Fusté-Escolano, 2002). Si bien no hay acuerdo sobre las variables a evaluar ni sobre los criterios de éxito, la medición a través de instrumentos estandarizados resulta básica para determinar la significación estadística o clínica y el tamaño del efecto de una intervención o tratamiento (Gómez-Penedo \& Roussos, 2012). Las mejorías más evidentes son las que se producen sobre los cambios sintomáticos. La utilización de medidas estandarizadas permite comparar el puntaje que obtiene un paciente con la población a la que pertenece y determinar el efecto de la intervención sobre el perfil sintomático, comparando su puntuación en diferentes momentos del proceso terapéutico. Usualmente, los clínicos se valen de entrevistas diagnósticas estructuradas y de diversas medidas estandarizadas para valorar la frecuencia y severidad sintomatológica. Según Clark y Beck (2012), estas últimas son de gran utilidad porque 
permiten detectar los síntomas más relevantes, brindan una medida de su gravedad, permiten su comparación con la población normativa y posibilitan su administración repetida a lo largo del curso del tratamiento.

\section{Alcances de la evaluación multidimensional en investigación clínica}

Clásicamente, cada sistema de respuesta se ha evaluado por separado, aplicándose en cada caso el método más idóneo (Fernández-Ballesteros, 1994). Sin embargo, esta metodología presentó diversas limitaciones que no justifican su evaluación por separado. Por un lado, aumentó la varianza intermétodo que hacía aumentar artificialmente la discordancia y desincronía entre sistemas de respuesta (Cano-Vindel \& Miguel-Tobal, 1990; Miguel-Tobal, 1990); por otro, no aportó información independiente que resulte significativa debido a la elevada correlación entre los instrumentos (Echeburúa, 1996). Es por ello que generalmente se opta por la aplicación de métodos menos costosos y fáciles de utilizar.

Tanto en la práctica clínica como en investigación, el autoinforme en su formato de escalas, cuestionarios e inventarios ha desplazado a los métodos conductuales clásicos (entrevista conductual y observación sistemática) y a los registros psicofisiológicos como instrumentos primordiales para la evaluación de los trastornos de ansiedad (Buela-Casal \& Sierra, 2004; Echeburúa, 1996; Fernández-Ballesteros, 1994; 2013c). Por ende, no solo han demostrado ser útiles para evaluar el sistema cognitivo, sino que también permiten obtener una primera aproximación a los sistemas fisiológicos y motores, lo que la impone como la técnica más empleada para determinar la intensidad sintomática y cuantificar los cambios terapéuticos (Buela-Casal \& Sierra, 2004; Cano-Vindel
\& Miguel-Tobal, 1990; Miguel-Tobal, 1990; Fernández-Ballesteros, 1994).

Por otro lado, si bien a partir de la década de 1960 se reconoció la necesidad de una evaluación conjunta de diversos sistemas de respuestas, a la hora de abordar el fenómeno de la ansiedad aún son escasas las investigaciones clínicas que la incluyen. Una revisión sistemática realizada por Cano-Vinel y Miguel-Tobal (1990) sobre la forma y los sistemas de evaluación en investigación clínica concluyó que, hasta entonces, existía un predominio de la evaluación de la dimensión cognitiva y motora sobre la fisiológica, principalmente a través de técnicas de autoinforme.

\section{Conclusión}

Este trabajo proporciona una descripción instrumental sobre la evaluación de la ansiedad y su evolución desde los primeros modelos psicofisiológicos de las emociones. Realiza un recorrido por las diversas técnicas y propuestas instrumentales adoptadas por los distintos modelos teóricos para evaluar cada dimensión. Repasa las aplicaciones clínicas y los alcances en investigación que posee la evaluación multidimensional, planteando la necesidad de una evaluación que logre abarcar la complejidad del constructo a fin de aumentar la eficacia de los tratamientos.

Según lo expuesto, existe un amplio repertorio instrumental para la evaluación multidimensional de la ansiedad con métodos específicos para medir cada una de sus dimensiones. Sin embargo, la valoración multimétodo ha planteado diversas limitaciones prácticas y metodológicas. Si bien las técnicas de autoinforme parecen ser la respuesta tecnológica más versátil y económica para obtener una primera aproximación a las dimensiones fisiológicas y conductuales, aún son escasos los instrumentos que incorporan dichas dimensio- 
nes, lo que crea una vacante en este aspecto. Es por ello que a la hora de evaluar la ansiedad se hace imperioso utilizar el método que permita la evaluación más completa posible del constructo teniendo en cuenta su multidimensionalidad.

\section{Referencias}

American Psychiatric Association (2013). DSM-5. Manual diagnóstico y estadístico de los trastornos mentales ( $5^{\mathrm{a}}$ ed.). Madrid, España: Médica Panamericana.

Bados-López, A., García-Grau, E., \& Fusté-Escolano, A. (2002). Eficacia y utilidad clínica de la terapia psicológica. International Journal of Clinical and Health Psychology, 2(3), 477-502. Recuperado de http:// www.aepc.es/ijchp/busca.php?coid=Espa\%F1ol

Barrios, B. A. (1988). On the changing nature of behavioral assessment. En A. S. Bellack \& M. Hersen (Eds.), Behavioral assessment: A practical handbook ( $3^{\mathrm{a}}$ ed., pp. 3-41). New York, NY: Pergamon.

Beck, A. T., Brown, G., Steer, R. A., Eidelson, J. I., \& Riskind, J. H. (1987). Differentiating anxiety and depression: A test of the cognitive content-specificity hypothesis. Journal of Abnormal Psychology, 96(3), 179-183. doi: 10.1037//0021-843x.96.3.179

Beck, A. T., Epstein, N., Brown, G., \& Steer, R. A. (1988). An inventory for measuring clinical anxiety: Psychometric properties. Journal of Consulting and Clinical Psychology, 56(6), 893-897. doi: 10.1037//0022-006x.56.6.893

Beck, J. S. (2000). Terapia cognitiva: Conceptos básicos y profundización. Barcelona, España: Gedisa.

Brown, T. A., Di Nardo, P. A., \& Barlow, D. H. (1994). Anxiety Disorders Interview for DSM-IV (ADIS-IV). San Antonio, TX: The Psychological Corporation.

Bruch, M., \& Meyer, V. (1996). La entrevista conductual. En G. B. Buela-Casal, V. E. Caballo \& J. C. Sierra (Eds.), Manual de evaluación en psicología clínica y de la salud (pp. 85-107). Madrid, España: Siglo XXI.

Buela-Casal, G., \& Sierra, J. C. (1997). Modelos de evalua- ción psicológica. En G. Buela-Casal \& J. C. Sierra (Eds.), Manual de evaluación psicológica. Fundamentos, técnicas y aplicaciones (pp. 103-145). Madrid, España: Siglo XXI.

Buela-Casal, G., \& Sierra, J. C. (2004). Manual de evaluación y tratamientos psicológicos. Madrid, España: Biblioteca Nueva.

Calero, M. D., \& Padilla, J. L. (2013). Técnicas psicométricas: Los test. En R. Fernández-Ballesteros (Ed.), Evaluación psicológica. Conceptos, métodos y estudio de casos (pp. 299-337). Madrid, España: Pirámide.

Calero, M. D., Márquez, M. O., Vizcarro, C., \& Fernández-Ballesteros, R. (2013). Otras técnicas: Objetivas y proyectivas. En R. Fernández-Ballesteros (Ed.), Evaluación psicológica. Conceptos, métodos y estudio de casos (pp. 275-298). Madrid, España: Pirámide.

Cannon, W. B. (1927a). The James-Lange's theory of emotion: A critical examination and an alteration. The American Journal of Psychology, 39(1-4), 106-124. doi: $10.2307 / 1415404$

Cannon, W. B. (1927b). Bodily changes in pain, hunger, fear and rage. New York and London: Appleton and Company.

Cano-Vindel, A. C., \& Miguel-Tobal, J. J. (1990). Revisión de la evaluación de los tres sistemas de respuesta de ansiedad en las terapias cognitivo-conductuales. Terapia del Comportamiento, 44-45, 109-114. Recuperado de: https://www.researchgate.net/publication/230577069_Revision_de_la_evaluacion_de_ los_tres_sistemas_de_respuesta_en_las_terapias_ cognitivo-conductuales

Carrobles, J. A., \& Buela-Casal, G. (1997). Técnicas psicofisiológicas. En G. Buela-Casal \& J. C. Sierra (Eds.), Manual de evaluación psicológica. Fundamentos, técnicas y aplicaciones (pp. 385-420). Madrid, España: Siglo XXI.

Castro-Solano, A. (2003). ¿Son eficaces las psicoterapias psicológicas? Psicodebate, 3, 59-90. doi: 10.18682/ pd.v3i0.503

Cautela, J. R., \& Upper, D. (1976). The behavioral inven- 
tory battery: The use of self-report measures in behavioral analysis and therapy. En M. Harsen \& A. S. Bellack (Eds.), Behavioral assessment: A practical handbook (pp. 76-109). Oxford, Reino Unido: Pergamon.

Clark, D. A., \& Beck, A. T. (2012). Terapia cognitiva para trastornos de ansiedad: Ciencia y práctica. Bilbao: Desclée de Brower.

De Ansorena-Cao, A., Cobo-Reinoso, J., \& Romero-Cagigal, I. (1983). El constructo ansiedad en psicología: Una revisión. Estudios de Psicología, 4(16), 31-45. doi: 10.1080/02109395.1983.10821366

Del Barrio, V. (1998). Evaluación de las características psicopatológicas. En R. Fernández-Ballesteros (Ed.), Introducción a la Evaluación Psicológica (Vol. II, pp. 120-159). Madrid, España: Pirámide.

Derogatis, L. R. (1977). Symptom Cecklist -90-Revised. PsycTESTS Dataset. doi: 10.1037/t01210-000

Echeburúa, E. (1996). Evaluación psicológica de los trastornos de ansiedad. En G. B. Buela-Casal, V. E. Caballo \& J. C, Sierra (Eds.), Manual de evaluación en psicología clínica y de la salud (131-160). Madrid: Siglo XXI.

Endler, N. S., Hunt, J. McV., \& Rosenstein, A. J. (1962). An SR inventory of anxiousness. Psychological Monographs: General and Applied, 76(17), 1-33. doi: 10.1037/h0093817

Eysenck, M. W., Derakshan, N., Santos, R., \& Calvo, M. G. (2007). Anxiety and cognitive performance: Attentional control theory. Emotion, 7(2), 336-353. doi: 10.1037/1528-3542.7.2.336

Eysenck, M. W. (1988). Anxiety and attention. Anxiety Research, 1(1), 9-15. doi: 10.1080/10615808808248216

Fernández-Ballesteros, R. (1994). Características básicas de la evaluación conductual. En R. Fernández-Ballesteros (Ed.), Evaluación conductual hoy, un enfoque para el cambio en psicología clínica y de la salud (pp. 85-110). Madrid, España: Pirámide.

Fernández-Ballesteros, R. (2013a). Conceptos y modelos básicos. En R. Fernández-Ballesteros (Ed.), Evaluación psicológica. Conceptos, métodos y estudio de casos (pp. 27-60). Madrid, España: Pirámide.

Fernández-Ballesteros, R. (2013b). La observación. En R. Fernández-Ballesteros (Ed.), Evaluación psicológica. Conceptos, métodos y estudio de casos (pp. 193233). Madrid, España: Pirámide.

Fernández-Ballesteros, R. (2013c). Los autoinformes. En R. Fernández-Ballesteros (Ed.), Evaluación psicológica. Conceptos, métodos y estudio de casos (pp. 235-274). Madrid, España: Pirámide.

First, M. B., Spitzer, R. L., Gibbon, M., \& Williams, J. B. (2004). Structured clinical interview for DSM-IV axis I disorders SCID-I: Clinician versión. Washington: American Psychiatric.

First, M. B., Spitzer, R. L., Gibbon, M., \& Williams, J. B. (2015). Structured clinical interview for DSM-5, SCID-5: Clinician version. Washington: American Psychiatric.

Gómez-Penedo, J. M., \& Roussos, A. (2012). ¿Cómo sabemos si nuestros pacientes mejoran? Criterios para la significancia clínica en psicoterapia: Un debate que se renueva. Revista Argentina de Clínica Psicológica, 21(2), 173-190. Recuperado de http://www.clinicapsicologica.org.ar/index.php

González-Martínez, M. T. (1993). Aproximación al concepto de ansiedad en psicología: Su carácter complejo y multidimensional. Aula, 5, 9-22. Recuperado de http://revistas.usal.es/index.php/0214-3402/index

Hamilton, M. (1959). The assessment of anxiety states by rating. British Journal of Medical Psychology, 32(1), 50-55. doi: 10.1111/j.2044-8341.1959.tb00467.x

Hayes, S. C., Nelson, R. O., \& Jarrett, R. B. (1986). Evaluating the quality of behavioral assessment. En R. O. Nelson \& S. C. Hayes, (Eds.), Conceptual foundations of behavioral assessment (pp. 463-503). New York, NY: Guilford Press.

Hollon, S. D., \& Kendall, P. C. (1980). Cognitive self-statements in depression: Development of an automatic thoughts questionnaire. Cognitive Therapy and Research, 4(4), 383-395. doi: 10.1007/bf01178214

James, W. (1884). What is an emotion? Mind, 9(34), 188205. doi: $10.1093 / \mathrm{mind} /$ os-ix.34.188 
James, W. (1890). The Principles of Psychology (Vol. I). New York, NY: Henry Holt. doi: 10.1037/10538-000

Jensen, J. B. (1996). Los cuestionarios de autoinforme en la evaluación conductual. En G. B. Buela Casal, V. E. Caballo, \& J. C. Sierra (Eds.), Manual de evaluación en psicología clínica y de la salud (pp. 109-127). Madrid: Siglo XXI.

Keegan, E., \& Holas, P. (2010). Cognitive-behavior therapy. Theory and practice. En Carlstedt, R. (Ed.), Handbook of integrative clinical psychology, psychiatry and behavioral medicine. Perspectives, practices and research (pp. 605-630). New York, NY: Springer.

Landis, C., \& Hunt, W. (1939). The startle pattern. Oxford, Reino Unido: Farrar \& Rinehart.

Lang, P. J., Bradley, M. M., \& Cuthbert, B. N. (1997). Motivated attention: Affect, activation, and action. En P. J. Lang, R. F. Simons \& M. Balaban (Eds.), Attention and orienting: Sensory and motivational processes (pp. 97-135). New York, NY: Routledge.

Lang, P. J., Melamed, B. G., \& Hart, J. (1970). A psychophysiological analysis of fear modification using an automated desensitization procedure. Journal of Abnormal Psychology, 76(2), 220-234. doi: 10.1037/ h0029875

Lange, C. G. (1885). The Emotions. Baltimore, MD: Williams and Wilkins.

Lecrubier, Y., Sheehan, D. V., Weiller, E., Amorim, P., Bonora, I., Sheehan, K. H., \& Dunbar, G. C. (1997). The Mini International Neuropsychiatric Interview (MINI). A short diagnostic structured interview: Reliability and validity according to the CIDI. European Psychiatry, 12(5), 224-231. doi: 10.1016/ s0924-9338(97)83296-8

Mandler, G., Mandler, J. M., \& Ulville, E. T. (1958). Autonomic feedback: The perception of autonomic activity. Journal of Abnormal \& Social Psychology, 56(3), 367-373. doi: 10.1037/h0048083

Márquez, M. O. (2013). La entrevista. En R. Fernández-Ballesteros (Ed.), Evaluación psicológica. Conceptos, métodos y estudio de casos (pp.167-192). Madrid, España: Pirámide.
Mathews, A., \& MacLeod, C. (1985). Selective processing of threat cues in anxiety states. Behaviour Research and Therapy, 23(5), 563-569. doi: 10.1016/0005-7967(85)90104-4

Menéndez-Carrillo, F. X., \& Maciá-Antón, D. (1994). Evaluación de los problemas de ansiedad. En R. Fernández-Ballesteros (Ed.), Evaluación conductual hoy, un enfoque para el cambio en psicología clínica y de la salud (pp. 426-483). Madrid, España: Pirámide.

Meyer, T. J., Miller, M. L., Metzger, R. L., \& Borkovec, T. D. (1990). Development and validation of the Penn State Worry Questionnaire. Behaviour Research and Therapy, 28(6), 487-495. doi: 10.1016/0005-7967(90)90135-6

Miguel-Tobal, J. J., \& Cano-Vindel, A. C. (1986). ISRA Inventario de situaciones y respuestas de ansiedad. Madrid: Tea.

Miguel-Tobal, J. J. (1990). La ansiedad. En J. L. Pinillos \& J. Mayor (Eds.), Tratado de Psicología General. Motivación y Emoción. Madrid, España: Alhambra Longman.

Mohlman, J., \& Gorman, J. M. (2005). The role of executive functioning in CBT: A pilot study with anxious older adults. Behaviour Research and Therapy, 43(4), 447465. doi: 10.1016/j.brat.2004.03.007

Öst, L. G., Jerremalm, A., \& Johansson, J. (1981). Individual response patterns and the effects of different behavioral methods in the treatment of social phobia. Behaviour Research and Therapy, 19(1), 1-16. doi: 10.1016/0005-7967(81)90107-8

Öst, L. G., Johansson, J., \& Jerremalm, A. (1982). Individual response patterns and the effects of different behavioral methods in the treatment of claustrophobia. Behaviour Research and Therapy, 20(5), 445-460. doi: 10.1016/0005-7967(82)90066-3

Pacheco-Unguetti, A. P., Lupiáñez, J., \& Acosta, A. (2009). Atención y ansiedad: Relaciones de la alerta y el control cognitivo con la ansiedad rasgo. Psicológica, 30(1), 1-25. Recuperado de https://www.uv.es/ psicologica

Pérez-Pareja, F. J. (1997). Autoinformes. En G. Buela-Casal 
\& J. C. Sierra (Eds.), Manual de evaluación psicológica. Fundamentos, técnicas y aplicaciones (pp. 297314). Madrid, España: Siglo XXI.

Quera, V., \& Behar, J. (1997). La observación. En G. Buela-Casal \& J. C. Sierra (Eds.), Manual de evaluación psicológica. Fundamentos, técnicas y aplicaciones (pp. 315-341). Madrid, España: Siglo XXI.

Sierra, J. C., Buela-Casal, G., \& Fernández, M. A. (2004). La entrevista clínica. En G. Buela-Casal \& J. C. Serra (Eds.), Manual de evaluación y tratamientos psicológicos (pp. 41-68). Madrid: Biblioteca Nueva.

Sierra, J. C., Ortega, V., \& Zubeidat, I. (2003). Ansiedad, angustia y estrés: Tres conceptos a diferenciar. Revista Mal-estar e Subjetividade, 3(1), 1059. Recuperado de http://pepsic.bvsalud.org/scielo. php?script $=$ sci_serial\&pid $=1518-6148$

Silva, F. (1998). La entrevista. En R. Fernández-Ballesteros (Ed.) Introducción a la Evaluación Psicológica (Vol I, pp. 252-278). Madrid, España: Pirámide.

Snaith, R. P., Baugh, S. J., Clayden, A. D., Husain, A., \& Sipple, M. A. (1982). The Clinical Anxiety Scale: An instrument derived from the Hamilton Anxiety Scale. British Journal of Psychiatry, 141(5), 518-523. doi: 10.1192/bjp.141.5.518

Spielberger, C. D., Gorsuch, R. L., \& Lushene, R. E. (1983). Manual for the State-Trait Anxiety Inventory. STAI (Form Y), Self evaluation questionnaire. Palo Alto, CA: Consulting Psychologists.

Tryon, W. W. (1997). Técnicas de registro de la actividad motora. En G. Buela-Casal \& J. C. Sierra (Eds.), Manual de evaluación psicológica. Fundamentos, técnicas y aplicaciones (pp. 369-384). Madrid, España: Siglo XXI.

Tyrer, P., Owen, R. T., \& Cicchetti, D. V. (1984). The brief scale for anxiety: A subdivision of the comprehensive psychopathological rating scale. Journal of Neurology, Neurosurgery \& Psychiatry, 47(9), 970-975. doi: 10.1136/jnnp.47.9.970

Vila, J. (1998). Evaluación psicofisiológica. En R. Fernández-Ballesteros (Ed.), Introducción a la Evaluación
Psicológica (Vol. II, pp. 335-370). Madrid, España: Pirámide.

Vila, J. (2004). Evaluación neurofisiológica. En V. del Barrio (Ed.), Evaluación psicológica aplicada a diferentes contextos. Madrid, España: UNED.

Vizcarro, C. (1998). Clasificación y criterios diagnósticos de la conducta anormal. En R. Fernández Ballesteros (Ed.), Introducción a la Evaluación Psicológica (Vol II, pp. 98-119). Madrid, España: Pirámide.

Watson, D., \& Clark, L. A. (1984). Negative affectivity: The disposition to experience aversive emotional states. Psychological Bulletin, 96(3), 465-490. doi: 10.1037//0033-2909.96.3.465

Watson, D., Clark, L. A., \& Tellegen, A. (1988). Development and validation of brief measures of positive and negative affect: The PANAS scales. Journal of Personality and Social Psychology, 54(6), 1063-1070. doi: 10.1037//0022-3514.54.6.1063

Wittchen, H. U., \& Boyer, P. (1998). Screening for anxiety disorders: Sensitivity and specificity of the Anxiety Screening Questionnaire (ASQ-15). The British Journal of Psychiatry, 173(S34), 10-17. doi: 10.1192/ s000712500029346x

Wolpe, J. (1977). Inadequate behavior analysis: The Achilles heel of outcome research in behavior therapy. Journal of Behavior Therapy and Experimental Psychiatry, 8(1), 1-3. doi: 10.1016/0005-7916(77)90095-7

Young, J. E. (1999). Cognitive therapy for personality disorders: A schema focused approach. ( $3^{\mathrm{a}} \mathrm{ed}$.). Sarasota, FL: Professional Resource.

Zigmond, A. S., \& Snaith R. P. (1983). The Hospital Anxiety and Depression Scale. Acta Psychiatrica Scandinavica, 67(6), 361-370. doi: 10.1111/j.1600-0447.1983. tb09716.x

Zung, W. W. K. (1971). A rating instrument for anxiety disorders. Psychosomatics, 12(6), 371-379. doi: 10.1016/s0033-3182(71)71479-0 